This is the accepted version of the article:

Bon I., Bartolí R., Cano-Sarabia M., de la Ossa N., de Vega V.M., Marín I., Boix J., Lorenzo-Zúñiga V.. Comparative study of electrical and rheological properties of different solutions used in endoscopic mucosal resection. Digestive Endoscopy, (2019). 31. : 276 - . 10.1111/den.13297.

Available at: https://dx.doi.org/10.1111/den.13297 


\title{
Comparative study of electrical and rheological properties of different solutions used in endoscopic mucosal resection
}

\author{
Ignacio Bon,1 Ramón Bartolí,3 Mary Cano-Sarabia,4 Napoleón de la Ossa,2 Vicente \\ Moreno de Vega, 1 Ingrid Marín, 1 Jaume Boix1,3 and Vicente Lorenzo-Zúñiga1,3
}

1Endoscopy Unit, and 2Department of Pathology, Germans Trias i Pujol University Hospital, 3 Network Biomedical Research Center of Hepathic and Digestive Diseases (CIBERehd), and 4 Catalan Institute of Nanoscience and Nanotechnology, CSIC and The Barcelona Institute of Science and Technology, Barcelona, Spain.

Background and Aim: The study of electrical and rheological properties of solutions to carry out endoscopic resection procedures could determinate the best candidate. An ex vivo study with porcine stomachs was conducted to analyse electrical resistivity $(\mathrm{R})$ and rheological properties (temperature, viscosity, height and lasting of the cushion) of different substances used in these techniques.

Methods: Tested solutions were: $0.9 \%$ saline (S), platelet-rich plasma (PRP), Gliceol (GC), hyaluronic acid $2 \%(H A)$, Pluronic-F127 20\% (PL), saline with $10 \%$ glucose (GS), Gelaspan (GP), Covergel-BiBio (TB) and PRP with TB (PRP+TB). Measurements of electrical and rheological properties were done at 0, 15, 30, 45 and 60 min after submucosal injection.

Results: Solutions showed a wide variability of transepithelial $\mathrm{R}$ after submucosal injection. Substances able to maintain the highest R 60 min post injection were TB, HA and PL. Protective solutions against deep thermal injury ( $T^{\underline{a}}$ lower than $\left.60^{\circ} \mathrm{C}\right)$ were $\mathrm{PL}\left(47.6^{\circ} \mathrm{C}\right)$, TB $\left(55^{\circ} \mathrm{C}\right)$ and $\mathrm{HA}\left(56.63^{\circ} \mathrm{C}\right)$. Shortest time to carry out resections were observed with GC (17.66), PRP (20.3) and GS (23.45). Solutions with less cushion decrease $(<25 \%)$ after 60 min were TB (11.74\%), PL (18.63\%) and PRP (22.12\%).

Conclusions: Covergel-BiBio, PL and HA were the best solutions with long-term protective effects (transepithelial R, lower thermal injury and less cushion decrease). Solutions with quicker resection time were GC, PRP and GS.

Key words: electrical property, endoscopic mucosal resection, endoscopic submucosal dissection, rheological property, submucosal injection

Corresponding: Vicente Lorenzo-Zúñiga, Endoscopy Unit, Department of Gastroenterology/CIBERehd, Hospital Universitari Germans Trias i Pujol, Carretera del Canyet s/n, 08916 Badalona, Spain. Email: vlorenzo.germanstrias@gencat.cat Received 20 July 2018; accepted 8 November 2018.

\section{INTRODUCTION}

\section{$\mathrm{D}$}

IFFERENT SOLUTIONS ARE widely used in endoscopic mucosal resection (EMR) or endoscopic submucosal

dissection (ESD) as submucosal injection to facilitate en bloc resection or to reduce the incidence of adverse events.1,2 The ideal solution with which to carry out submucosal injection must contribute to a better clinical outcome. This solution should achieve and maintain a long lasting cushion to safely allow the physician to carry out the required techniques, be non-toxic so as not to influence histological evaluation and should be an inexpensive, readily available and easily administered solution.2-7 Endoscopic resection techniques are carried out using an electrosurgical unit (ESU) which works by applying an electrical current through an electrode in contact with the tissue and, depending on the setting of the machine, carrying out coagulation or cutting. The electric circuit that forms between the ESU, the electrode and the tissue can be affected by many variables, influencing the outcome of the procedure. Thus, electrical characteristics of the submucosal injection solution used also have an effect on this outcome.8 Hence, measurement of electrical resistivity (i.e. the fundamental property of a material that quantifies how strongly that material opposes the flow of electric current), viscosity or osmolarity are of great importance for the outcome of the procedure. Therefore, we aim to analyse electrical (resistivity) and rheological properties (temperature, viscosity, height and lasting of the cushion) of different solutions to carry out EMR in an ex vivo model of porcine stomachs. 


\section{METHODS}

\section{$\mathrm{F}$}

RESH PORCINE STOMACHS were used to analyse submucosal injection solution properties. Measurements

were carried out in the fundus. Each solution $(2 \mathrm{~mL})$ was injected using a $22 \mathrm{G}$ needle. For measurement of cushion decrease and transepithelial resistance, stomach portions of $5 \mathrm{~cm}$ were used. Stomach portions were maintained at $37^{\circ} \mathrm{C}$ during the test. EMR resection was carried out after prior submucosal injection with a standard polypectomy snare (Olympus Medical Systems, Tokyo, Japan) with blended current on full stomachs for the measurement of EMR time and muscle temperature. To standardize the same force applied to the polypectomy snare in all procedures, the snare was placed in a laboratory syringe pump (Advance Infusion Pump Series 1200; Parkland Scientific, Coral Springs, FL, USA) with a linear force of $10000 \mathrm{~g}$.

\section{Solutions}

The following solutions were tested: $0.9 \%$ saline (S), platelet-rich plasma (PRP), Gliceol (GC), hyaluronic acid 2\% (HA), Pluronic-F127 20\% (PL), saline with 10\% glucose (GS), Gelaspan (GP), Covergel-BiBio (TB) and PRP with TB (PRP+TB). Saline, GS and GP were acquired from the hospital pharmacy service (Germans Trias i Pujol University Hospital, Barcelona, Spain). GC was manufactured in our laboratory (Helath Research Institute Germans Trias i Pujol, Barcelona, Spain). PRP was obtained by extraction of blood with a syringe containing 1/10 total blood volume of $10 \%$ sodium citrate. Then, blood was centrifuged at $160 \mathrm{~g}$ for $20 \mathrm{~min}$ at room temperature; this resulted in a two-phases tube, the top one containing plasma. Plasma was recollected and transferred to a new centrifuge tube, and further centrifuged at $400 \mathrm{~g}$ for $15 \mathrm{~min}$. The result of this was platelet-poor plasma (2/3 of total volume) and PRP (the bottom $1 / 3$ of the total volume). PRP was activated by addition of $0.05 \mathrm{~mL} / 1 \mathrm{~mL}$ PRP of $10 \%$ calcium chloride. Covergel-BiBio is a mixture of four components9: Pluronic-F127 modified to add acrylate motifs for its irreversible gelation, hyaluronic acid (TCI Europe, Zwijndrecht, Belgium), methylcellulose (Sigma, St Louis, MO, USA), Rifaximine (Sigma) and Irgarcure 2959 as a photoinitiator (Sigma).

\section{Evaluation of electrical and rheological properties}

Electrical resistivity or resistance $(R)$ of materials to the flow of an electric current was evaluated using a multimeter SK-7707 (KAISE Corporation, Ueda City, Japan) with conventional electrodes; one electrode was positioned at the apical surface of the cushion (gastric mucosa) and the other on the basal surface of the cushion (serosa layer), to evaluate the transepithelial $\mathrm{R}$ that the stomach wall had with the submucosal solution injected. Measurements were done at $0,15,30,45$ and 60 min after submucosal injection (Fig. 1). Temperature ( $\mathrm{T}^{\mathrm{a}}$ ) analysis was carried out using a thermometer K/J type thermocouple (Uxcell, Kwai Fong, Hong Kong) with the sensor located at the muscle layer to check the risk of deep thermal injury (Fig. 2). Measurement started after submucosal injection and finished after EMR was done. Moreover, time needed to carry out EMR was registered with each solution. Submucosal cushion height was studied taking into account three different aspects: differences of height at time 0 after injection, differences of height at time $60 \mathrm{~min}$ after injection and the percentage of decrease for each substance. We evaluated this by taking standardized pictures at times: 0, 15, 30, 45 and $60 \mathrm{~min}$. Images were evaluated using ImageJ software (NIH, Bethesda, MD, USA). Decrease of the cushion is shown as \% of the original height of each solution. All determinations were carried out in triplicate to calculate mean data. Osmolarity and viscosity of each solution were obtained from each manufacturer.

\section{Statistical analysis}

A descriptive analysis was carried out for each experiment. ANOVA tests were carried out to compare groups. Tuckey tests were done as post-hoc analysis. SPSS software, 15.0 version was used). A nonparametric Spearman correlation study was carried out between all the characteristics evaluated using SPSS software, 15.0 version software.

\section{RESULTS}

\section{Electrical and rheological properties}

$\mathrm{T}$

RANSEPITHELIAL RWAS measured at times $0,15,30,45$ and $60 \mathrm{~min}$ after substance injection on the

submucosal layer. Resistivity of porcine stomach without submucosal injection was $5 \times 10^{4} \Omega$. Solutions showed a wide variability of transepithelial R after submucosal injection (time 0 : GP $20 \times 10^{4} \Omega$, TB $20 \times 10^{4} \Omega$, GC $16 \times 10^{4} \Omega$, GS $13 \times 10^{4} \Omega$, HA $9 \times 10^{4} \Omega$, PL $8 \times 10^{4} \Omega$, PRP $6.5 \times 10^{4} \Omega$, S $6 \times 10^{4} \Omega$, and PRP+TB $6 \times 10^{4} \Omega$; Table 1). Next, measurements at 15,30 and 45 min showed a trend to decrease $R$ in all solutions except HA, PL, PRP and PRP+TB, which maintained stable values. Substances that were able to maintain a higher $R$ after $60 \mathrm{~min}$ post injection were TB (7x10 $\Omega)$, HA $\left(7 \times 10^{4} \Omega\right)$ and PL $\left(7 \times 10^{4} \Omega\right)$. Values represent the mean of three separate experiments for each substance (Fig. $3)$. Endoscopic mucosal resection without any solution used to create a cushion resulted in a muscle layer $T^{\mathrm{a}}$ of $79.48^{\circ} \mathrm{C}$ and total time for resection of $31 \mathrm{~s}$ (basal determinations). Solutions showed very different patterns in $\mathrm{T}^{\mathrm{a}}$ increase during EMR at the muscle layer (Fig. 4). High risk of deep thermal injury $\left(\mathrm{T}^{\mathrm{a}}\right.$ higher than $60^{\circ} \mathrm{C}$ ) was 
observed with S $\left(198.4^{\circ} \mathrm{C}\right)$, GP $\left(133.55^{\circ} \mathrm{C}\right)$, PRP $\left(110.4^{\circ} \mathrm{C}\right)$, GS $\left(82.85^{\circ} \mathrm{C}\right), \mathrm{GC}\left(82.4^{\circ} \mathrm{C}\right)$ and PRP+TB $\left(80.1^{\circ} \mathrm{C}\right)$. In contrast, protective solutions were $\mathrm{PL}\left(47.6^{\circ} \mathrm{C}\right), \mathrm{TB}\left(55^{\circ} \mathrm{C}\right)$ and $\mathrm{HA}\left(56.63^{\circ} \mathrm{C}\right)$. Time to carry out EMR was comparable or higher than basal with HA (28" ), GP (30.73" ), PRP+TB (33" ), S (33.41" ), PL (34.64" ) and TB (51.67). Solutions able to shorten this time were GC (17.66" ), PRP (20.3" ) and GS (23.45" ) (Fig. 5). Submucosal cushion height with the different solutions at time 0 and $60 \mathrm{~min}$ after injection is shown in Table 1 . Substances capable of maintaining at least $75 \%$ of the original height after 1 hour were (cushion decrease <25\%): TB (11.74\%), PL (18.63\%) and PRP (22.12\%) (Fig. 6). Osmolarity and viscosity of each solution are shown in Table 1. A nonparametric Spearman's correlation study was done for all the characteristics evaluated and results are shown in

Table 2. Correlation quantifies the degree to which two variables are related. Correlation tests compute a correlation coefficient $(r)$ that shows how much one variable tends to change when the other variable changes. When $r$ is 0.0 , there is no relationship. When $r$ is positive, there is a trend that one variable goes up as the other one goes up. When $r$ is negative, there is a trend that one variable goes up as the other one goes down. Statistical correlation study showed a significant correlation only between: resistivity of the solutions and the muscular temperature reached after EMR ((0.7851 P-value 0.002, negative correlation, the higher the resistivity, the lower the temperature reached); resistivity of the solutions and the time to carry out EMR (0.6938 P-value 0.042, positive correlation, the higher the resistivity, the longer the time to carry out EMR); and between viscosity and the muscle temperature reached after EMR (0.8740 Pvalue 0.003, negative correlation, the higher the viscosity the lower the temperature reached). The rest of the correlations, although not statistically significant, show a clear trend. For example, the risk of thermal injury seems to be higher with higher osmolarity, and EMR time is higher with higher resistivity, viscosity and osmolarity of the substance.

\section{DISCUSSION}

$\mathrm{E}$ NDOSCOPIC MUCOSAL RESECTION and ESD have been established as safe and feasible techniques for the removal of large lesions throughout the gastrointestinal tract. Avoiding the main complications associated with bleeding and perforation is of interest in the field of endoscopy and, to achieve this, many improvements in resection techniques have been developed. Submucosal injection, as the introduction of a substance into the submucosal layer of the gastrointestinal wall to elevate the mucosal layer, thus protecting the muscle layer, has been proven effective to prevent these complications and to improve the clinical outcomes of the procedure. The search for the most appropriate solution to carry out this submucosal injection is still a matter of debate. Studies have been focused on the capacity of the solution to elevate the mucosa and to maintain this elevation, ignoring some other important aspects of these solutions that may have an important role on the outcome of the procedure. Endoscopic resections are carried out using an ESU which applies an electrical current to carry out the desired coagulation or cutting for the procedure; thus, studying the electrically related properties of the solutions is of great interest.4,6-8 In the present study, we have tested both commercial and non-commercial solutions to carry out submucosal injection and we have tried to connect electrical resistivity, viscosity and osmolarity of the solutions with clinical aspects that will have an impact on the clinical outcome, such as height and duration of the elevation, time to carry out EMR or temperature reached after EMR. Resistivity is a property of a material that quantifies the opposition of said material to the flow of an electric current. Osmolarity is the measure of a solute concentration, the number of osmoles of solute particles per unit of volume in a solution. This value is indicative of the osmotic pressure of a solution which will determine how this solution will diffuse across a semipermeable membrane (osmosis). Viscosity is often referred to as the thickness of a fluid and, at a molecular level, it is a result of the interaction between the different molecules in a fluid and it will determine the energy needed to make a fluid flow. Previous studies related the electrical resistivity of blood to its viscosity, affected by the quantity of fibrinogen.10,11

Our results showed wide variability of transepithelial after submucosal injection. Substances with high viscosity were associated with higher and maintained stable values of R (TB, PL and HA). These solutions also have a higher protective effect against deep thermal injury and less cushion decrease. In contrast, solutions able to carry out EMR in a shorter time were GC, PRP and GS. Statistical correlation study showed a significant correlation only between: resistivity of the solutions and the muscle temperature reached after EMR, resistivity of the solutions and the time to carry out EMR and between viscosity and the muscle temperature reached after EMR.

Our study was an attempt to explore electrical and rheological properties of different solutions used in EMR. An ex vivo model to study any organ physiology has some drawbacks such as absence of perfusion and active absorption that, in this case, can mean a longer time for the submucosal fluid to resolve; nonetheless, we tried to minimize this problem by using porcine stomachs within the first hour after the animal's death, which avoids significant tissue changes.12 In the present study, we tried to standardize all procedures as much as possible by using the same part of the stomach for each substance, creating a $2-\mathrm{mL}$ submucosal cushion, applying the same force to the polypectomy snare by using a laboratory syringe pump at a set value and cutting the same amount of stomach mucosal layer. The small-sized mucosal resections we carried out do not usually require a submucosal injection clinically as lesions smaller than $2 \mathrm{~cm}$ do not require submucosal injection for EMR or, if it is done, the solution used does not have much 
impact on the final outcome.7 Nonetheless, we prioritized standardization of the lesions and the EMR technique for better comparison of the results.

We also note our small size sample and the lack of in vivo data, as this was a proof of concept research work in which we wanted to see a possible correlation or trend between the characteristics studied and, thus, open the door for a future in vivo study that is more clinically representative. In conclusion, HA, PL and TB were the best solutions with long-term protective effects (transepithelial, lower thermal injury and less cushion decrease). Solutions with quicker resection time were GC, PRP and GS.

\section{CONFLICTS OF INTEREST}

$\mathrm{V}$

LORENZO -Zúnñiga, R. Bartolí and J. Boix declare patent authorship of one of the tested solutions

(Covergel-BiBio). Other authors have no conflicts of interest to disclose. The authors who have taken part in this study do not have a relationship with the manufacturers of the drugs involved either in the past or present and did not receive funding from the manufacturers to carry out their research.

\section{REFERENCES}

1 Conio M. Endoscopic mucosal resection. Gastroenterol. Hepatol.2011; 7: 248-50.

2 Ferreira A, Moleiro J, Torres J, Dinis-Ribeiro M. Solutions for submucosal injection in endoscopic resection: a systematic review and meta-analysis. Endosc. Int. Open. 2015; 04:E1-16.

3 Polymeros D, Kotsalidis G, Triantafyllou K, Karamanolis G, Panagiotides JG, Ladas SD. Comparative performance of novel solutions for submucosal injection in porcine stomachs: an ex vivo study. Dig. Liver Dis. 2010; 42: 226-9.

4 Huai ZY, Xian WF, Jiang LC, Chen WX. Submucosal injection solution for endoscopic resection in gastrointestinal tract: a traditional and network meta-analysis. Gastroenterol. Res. Pract. 2015; 2015: https://doi.org/10.1155/2015/702768.

5 Uraoka T, Saito Y, Yamamoto K, Fujii T. Submucosal injection solution for gastrointestinal tract endoscopic mucosal resection and endoscopic submucosal dissection. Drug Des. Devel. Ther. 2008; 2: 131-8.

6 Fujishiro M, Yahagi N, Kashimura K et al. Tissue damage of different submucosal injection solutions for EMR. Gastrointest. Endosc. 2005; 62: 933-42.

7 Yandrapu H, Desai M, Siddique S et al. Normal saline solution versus other viscous solutions for submucosal injection during endoscopic mucosal resection: a systematic review and metaanalysis. Gastrointest. Endosc. 2017; 85: 693-9.

8 Park S, Chun HJ, Kim CY et al. Electrical characteristics of various submucosal injection fluids for endoscopic mucosal resection. Dig. Dis. Sci. 2008; 53: 1678-82.

9 Lorenzo-Zúñiga V, Boix J, Moreno de Vega V, Bon I, Marín I, Bartolí. R. Endoscopic shielding technique with a newly developed hydrogel to prevent thermal injury in two experimental models. Dig. Endosc. 2017; 29: 702-11.

10 Pop GA, de Backer TL, de Jong $\mathrm{M}$ et al. On-line electrical impedance measurement for monitoring blood viscosity during onpump heart surgery. Eur. Surg. Res. 2004; 36: 259-65.

11 Zhao TX, Jacobson B. Quantitative correlations among fibrinogen concentration, sedimentation rate and electrical impedance of blood. Med. Biol. Eng. Comput. 1997; 35: 181-5.

12 Søndenaa K, Kjellevold KH. A prospective study of the length of the distal margin after low anterior resection for rectal cancer. Int. J. Colorectal Dis. 1990; 5: 103-5. 
Table 1 Electrical and rheological properties of different solutions used to carry out submucosal injection

\begin{tabular}{lcccccccc}
\hline Solutions & $\begin{array}{c}\text { Resistivity } \\
\left(10^{4} \Omega\right)\end{array}$ & $\begin{array}{c}T^{\mathrm{a}} \text { muscle } \\
\left({ }^{\circ} \mathrm{C}\right)\end{array}$ & $\begin{array}{c}\text { Heigh } \mathrm{t}=0 \\
(\mathrm{~cm})\end{array}$ & $\begin{array}{c}\text { Heigh } \mathrm{t}=60 \\
(\mathrm{~cm})\end{array}$ & $\begin{array}{c}\text { Cushion } \\
\text { decrease }(\%)\end{array}$ & $\begin{array}{c}\text { Time } \\
(\mathrm{s})\end{array}$ & $\begin{array}{c}\text { Osmolarity } \\
(\mathrm{mOsm} / \mathrm{L})\end{array}$ & $\begin{array}{c}\text { Viscosity } \\
(\mathrm{Pa})\end{array}$ \\
\hline Saline & 6 & 198.4 & 1.68 & 1.22 & 27.46 & 33.4 & 286 \\
PRP & 6.5 & 110.4 & 2.14 & 1.67 & 22.12 & 20.3 & 278 & 0.0043 \\
Gliceol & 16 & 82.4 & 2.12 & 1.53 & 28.74 & 17.6 & 288 & 0.01 \\
HA & 9 & 56.6 & 2.41 & 1.76 & 27.06 & 28 & 282 & 0.04 \\
PL & 8 & 47.6 & 2.37 & 1.93 & 18.63 & 34.6 & 300 & 0.9 \\
GS & 13 & 82.9 & 2.33 & 1.15 & 50.84 & 23.5 & 556 & 0.008 \\
Gelaspan & 20 & 133.6 & 2.45 & 1.73 & 30.51 & 30.7 & 284 & 0.009 \\
TB & 20 & 55 & 2.63 & 2.32 & 11.74 & 51.7 & 300 & 0.9 \\
PRP+TB & 6 & 80.1 & 2.60 & 1.65 & 36.55 & 33 & 280 \\
\hline
\end{tabular}

PRP, platelet-rich plasma; HA, hyaluronic acid 2\%; PL, pluronic-F127 20\%; GS, saline with 10\% glucose; TB, Covergel-BiBio.

Table 2 Nonparametric Spearman's correlation for all characteristics evaluated

\begin{tabular}{lcccccc}
\hline & Resistivity & Viscosity & Osmolarity & Muscle $T^{\star}$ & EMR time & Cushion decrease \\
\hline Resistivity & - & 0.6352 & 0.3575 & $-0.7851^{\star \star}$ & $0.6938^{\star}$ & -0.5295 \\
Viscosity & 0.6352 & - & -0.3376 & $-0.8740^{\star \star}$ & 0.4958 & -0.6051 \\
Osmolarity & 0.3575 & -0.3376 & - & 0.02510 & 0.3904 & 0.07531 \\
Muscle T & $-0.7851^{\star \star}$ & $-0.8740^{\star \star}$ & 0.02510 & - & -0.3833 & 0.4833 \\
EMR time & $0.6938^{\star}$ & 0.4958 & 0.3904 & -0.3833 & - & -0.4500 \\
Cushion decrease & -0.5295 & -0.6051 & 0.07531 & 0.4833 & -0.4500 & - \\
\hline
\end{tabular}

*P-value $<0.05$.

$\star *$ P-value $<0.01$.

EMR, endoscopic mucosal resection.

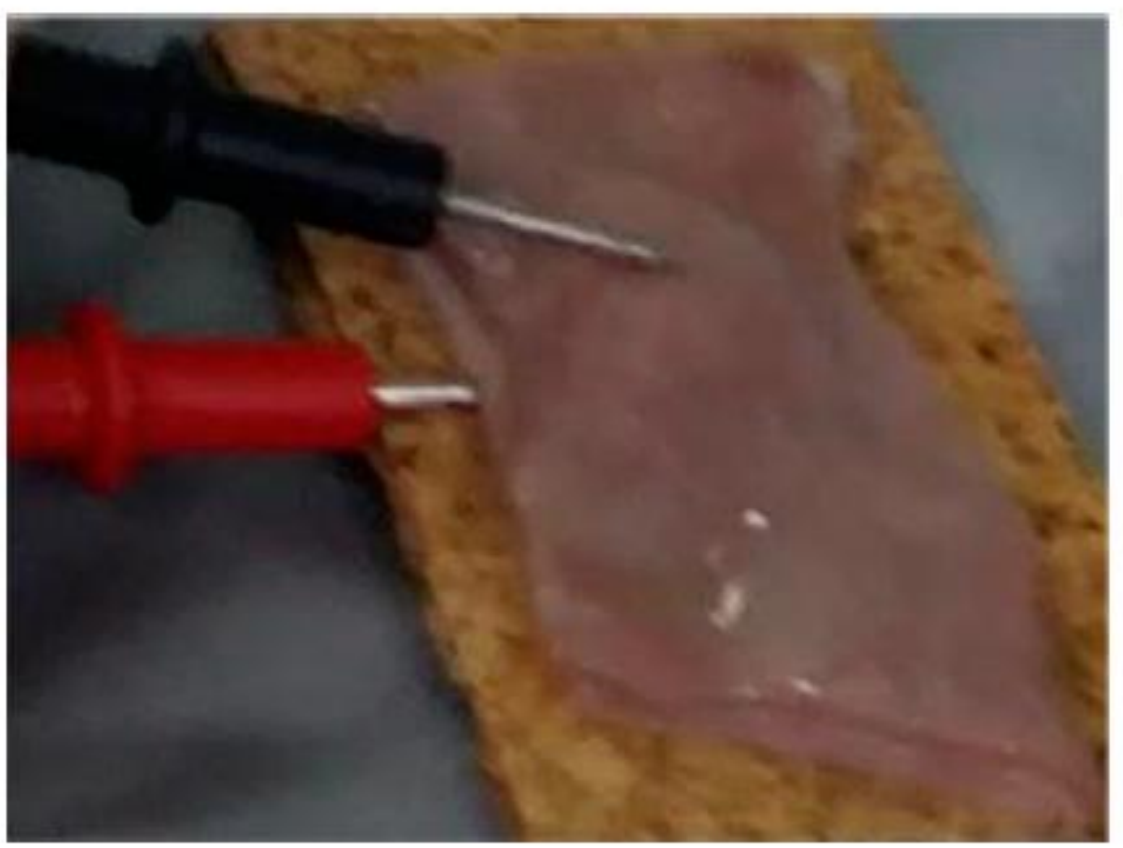

Figure 1 Evaluation of electrical resistivity with a multimeter. 


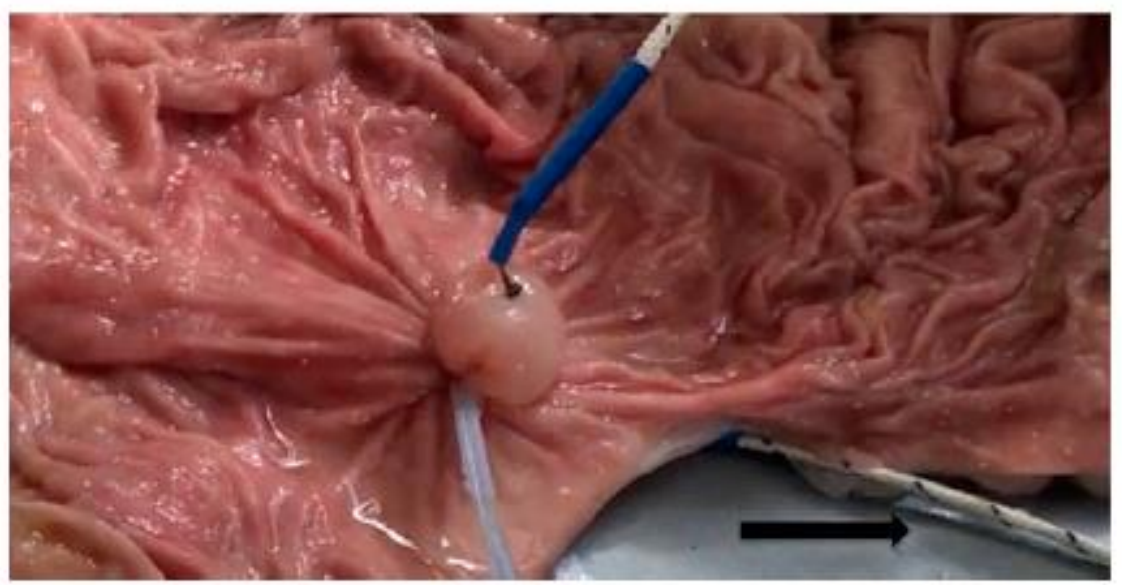

Figure 2 Analysis of temperature during endoscopic mucosal resection with a thermometer on the apical face of the stomach and the other (black arrow) on the serosa layer.
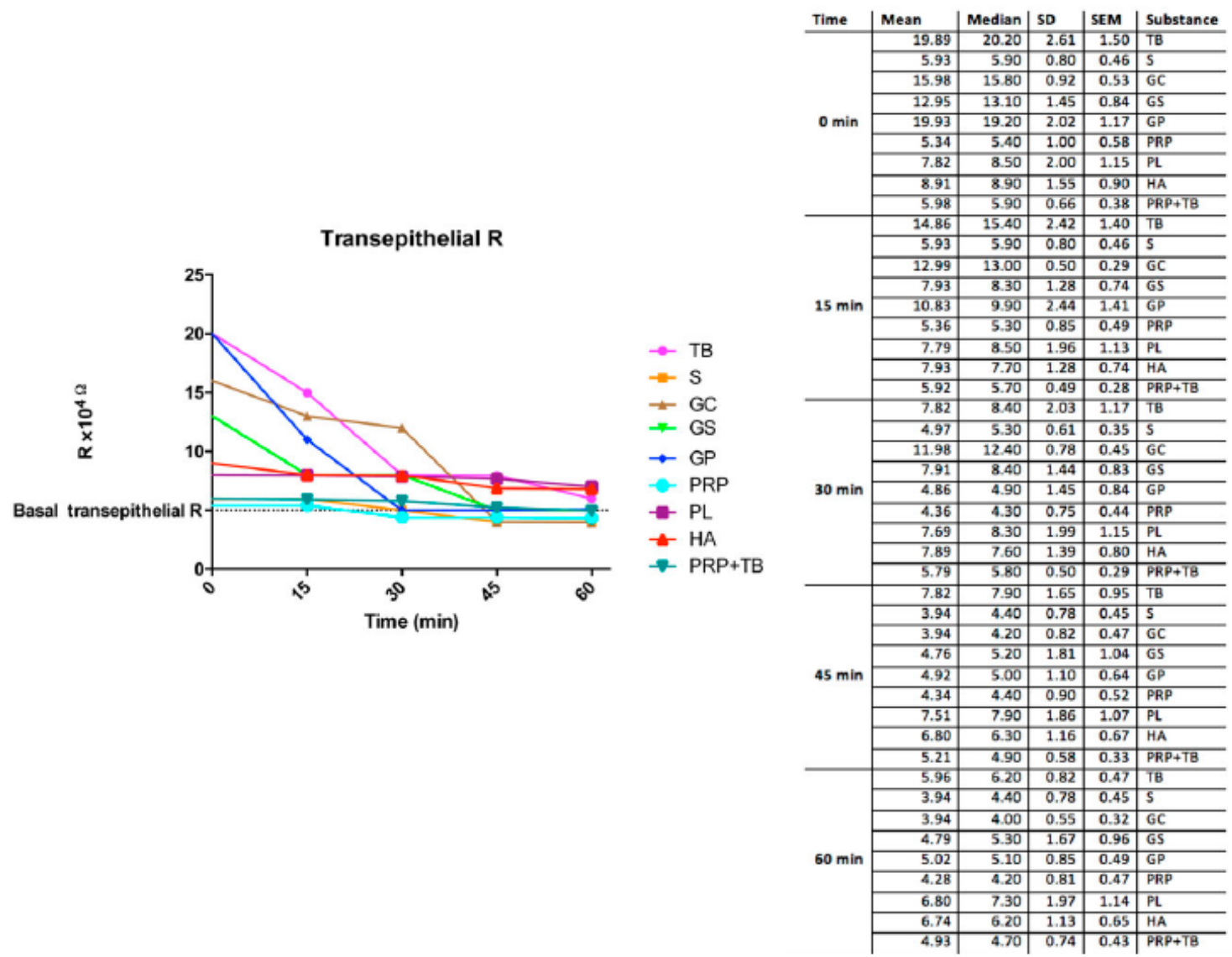

Figure 3 Transepithelial resistivity $(R)$ of different solutions in fresh porcine stomach at baseline, 15, 30, 45 and 60 min after submucosal injection. Solutions used were platelet-rich plasma (PRP), 0.9\% saline (S), Gliceol (GC), Pluronic-F127 20\% (PL), hyaluronic acid $2 \%(\mathrm{HA})$, saline with $10 \%$ glucose (GS), Gelaspan (GP), Covergel-BiBio (TB) and PRP+TB. 


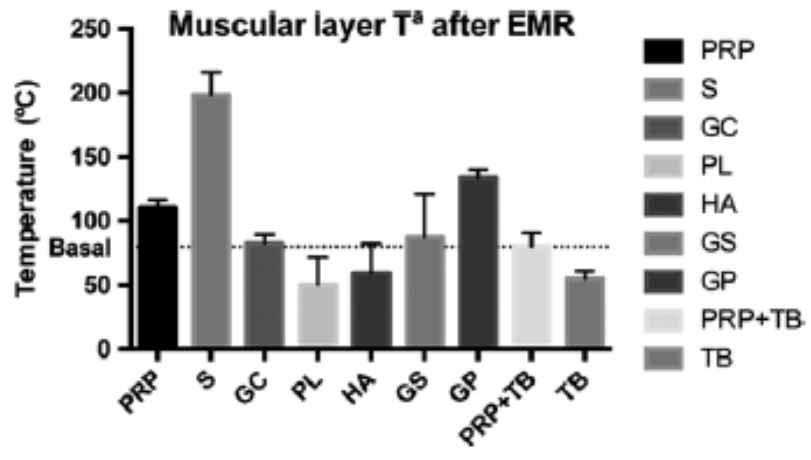

Figure 4 Temperature after endoscopic mucosal resection (EMR) reached muscular layer. Values are the mean of three separate experiments for each substance. Solutions used were platelet-rich plasma (PRP), 0.9\% saline (S), Gliceol (GC), Pluronic-F127 20\% (PL), hyaluronic acid $2 \%$ $(\mathrm{HA})$, saline with $10 \%$ glucose (GS), Gelaspan (GP), CovergelBiBio (TB) and PRP+TB.

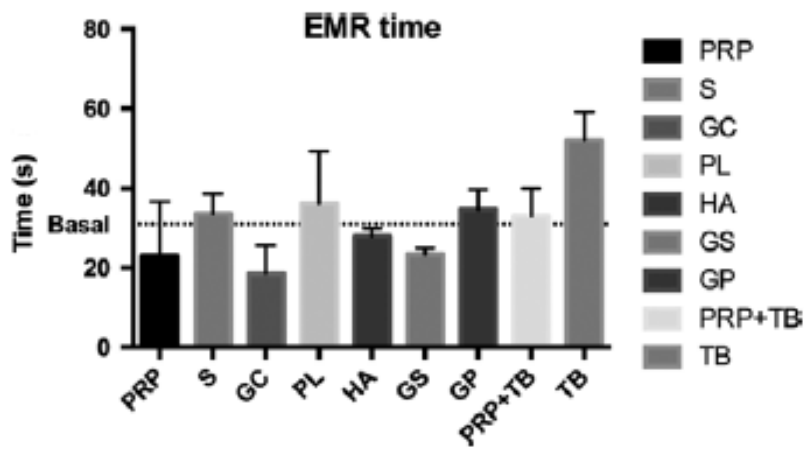

Figure 5 Time required to carry out endoscopic mucosal resection (EMR) with different substances using a standard polypectomy snare. Values are the mean of three separate experiments for each substance. Solutions used were platelet-rich plasma (PRP), $0.9 \%$ saline (S), Gliceol (GC), Pluronic-F127 20\% (PL), hyaluronic acid 2\% (HA), saline with $10 \%$ glucose (GS), Gelaspan (GP), Covergel-BiBio (TB) and PRP+TB. 


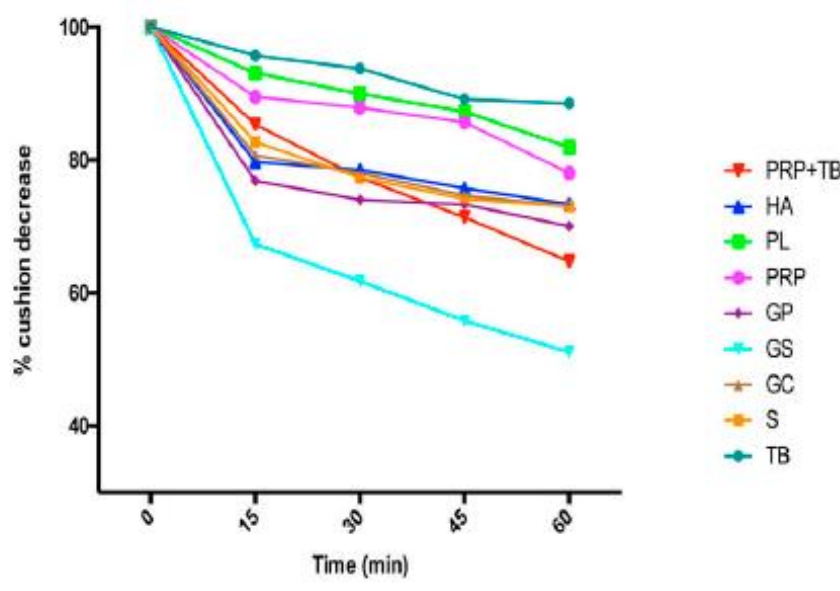

\begin{tabular}{|c|c|c|c|c|c|}
\hline Time & Mean & Median & SD & SEM & Substance \\
\hline \multirow{9}{*}{$15 \mathrm{~min}$} & 76.67 & 74.20 & 7.05 & 4.07 & GP \\
\hline & 95.63 & 97.08 & 5.05 & 2.91 & TB \\
\hline & 82.67 & 82.27 & 2.80 & 1.62 & $s$ \\
\hline & 80.11 & 83.55 & 11.49 & 6.63 & $\mathrm{GC}$ \\
\hline & 66.46 & 68.68 & 13.20 & 7.62 & GS \\
\hline & 85.26 & 85.66 & 4.57 & 2.64 & PRP+TB \\
\hline & 89.06 & 90.28 & 10.88 & 6.28 & PRP \\
\hline & 92.98 & 95.08 & 5.37 & 3.10 & PL \\
\hline & 79.41 & 83.02 & 8.07 & 4.66 & HA \\
\hline \multirow{9}{*}{$30 \mathrm{~min}$} & 73.28 & 66.39 & 13.30 & 7.68 & GP \\
\hline & 93.59 & 97.08 & 6.51 & 3.76 & TB \\
\hline & 77.00 & 79.46 & 9.29 & 5.36 & $S$ \\
\hline & 77.06 & 81.34 & 14.80 & 8.55 & $\mathrm{GC}$ \\
\hline & 60.33 & 61.92 & 16.12 & 9.30 & GS \\
\hline & 77.27 & 75.88 & 4.15 & 2.39 & $\mathrm{PRP}+\mathrm{TB}$ \\
\hline & 87.41 & 87.97 & 11.04 & 6.38 & PRP \\
\hline & 89.88 & 90.20 & 4.27 & 2.46 & PL \\
\hline & 78.25 & 82.02 & 7.88 & 4.55 & HA \\
\hline \multirow{9}{*}{$45 \mathrm{~min}$} & 72.48 & 65.24 & 14.11 & 8.15 & GP \\
\hline & 88.96 & 89.45 & 7.10 & 4.10 & TB \\
\hline & 73.66 & 74.57 & 10.13 & 5.85 & 5 \\
\hline & 72.89 & 80.67 & 19.06 & 11.01 & $G C$ \\
\hline & 53.96 & 54.93 & 17.57 & 10.14 & GS \\
\hline & 70.87 & 72.38 & 10.44 & 6.03 & $P R P+T B$ \\
\hline & 85.21 & 86.11 & 10.84 & 6.26 & PRP \\
\hline & 87.18 & 85.46 & 5.51 & 3.18 & $\mathrm{PL}$ \\
\hline & 75.45 & 79.20 & 7.78 & 4.49 & HA \\
\hline \multirow{9}{*}{$60 \mathrm{~min}$} & 69.49 & 64.36 & 11.30 & 6.52 & GP \\
\hline & 88.26 & 87.99 & 8.54 & 4.93 & $T B$ \\
\hline & 72.54 & 73.15 & 10.16 & 5.87 & 5 \\
\hline & 71.26 & 78.93 & 18.90 & 10.91 & GC \\
\hline & 49.16 & 49.28 & 17.19 & 9.92 & GS \\
\hline & 63.45 & 67.19 & 15.42 & 8.91 & PRP+TB \\
\hline & 77.88 & 75.98 & 6.14 & 3.55 & PRP \\
\hline & 81.37 & 82.40 & 11.64 & 6.72 & $\mathrm{PL}$ \\
\hline & 72.94 & 78.67 & 9.54 & 5.51 & HA \\
\hline
\end{tabular}

Figure 6 Percentage of submucosal cushion decrease of the height acquired by each substance at time 0 . Values are the mean of three separate experiments for each substance. Solutions used were platelet-rich plasma (PRP), $0.9 \%$ saline (S), Gliceol (GC), Pluronic-F127 20\% (PL), hyaluronic acid $2 \%(\mathrm{HA})$, saline with $10 \%$ glucose (GS), Gelaspan (GP), Covergel-BiBio (TB) and PRP+TB. 